\title{
Effect of Location-Awareness on Rendezvous Behaviour
}

\author{
David Dearman, Kirstie Hawkey and Kori M. Inkpen \\ Faculty of Computer Science, Dalhousie University \\ Halifax, NS B3H 1W5 \\ \{dearman, hawkey, inkpen $\}$ cs.dal.ca
}

\begin{abstract}
This paper presents an exploratory field study investigating the behavioral effects of mobile location-aware computing on rendezvousing. Participants took part in one of three mobile device conditions (a mobile phone, a location-aware handheld or both a mobile phone and a location-aware handheld) and completed different rendezvousing scenarios. We present one of the scenarios in depth and discuss the effect of location-awareness on rendezvous behaviour.
\end{abstract}

\section{Author Keywords}

CSCW, location-aware computing, mobile devices, rendezvousing

\section{ACM Classification Keywords}

H.5.3. Group and Organization Interfaces: Collaborative Computing.

\section{INTRODUCTION}

Mobile phones have transformed our social interactions and behaviours. They provide a rich verbal communication channel that enables us to exchange contextual information. However, location is difficult to convey accurately through dialogue, resulting in ambiguity and misunderstandings. Location information provides an obvious benefit for rendezvousing; however, it is unclear how this information will affect social behaviours. Location-awareness is fundamentally different from active verbal communication and may alter people's actions while rendezvousing.

Projects using precise location awareness include an location-aware event planner [6] and ActiveCampus [3]. These systems provide visual location-awareness of all collaborators and an active communication channel (e.g. text messaging, voice). WatchMe [5] builds upon locationawareness, providing additional contextual information by comparing user movements to previous patterns terminating at user-defined locations. The context of the user's location is displayed descriptively (e.g. "gym") rather than with absolute coordinates or map annotations.

The main goal of our research is to investigate how location-aware technology impacts rendezvousing behaviour (people coming together at an agreed upon time and location). Group behaviours related to rendezvousing have been explored [1,2] through detailed diary studies

Copyright is held by the author/owner(s).

CHI 2005, April 2-7, 2005, Portland, Oregon, USA.

ACM 1-59593-002-7/05/0004. whose results illustrate common rendezvousing behaviours and challenges. An investigation of technology to support rendezvousing (mobile phones, text messaging, email, and voicemail) demonstrated that mobile phones are the current preferred method of communication [2]. When mobile, people often initiate a rendezvous by first agreeing upon a general time and place and then refining the location and time through subsequent messages [4].

This paper presents a field study exploring the use of mobile phones and location-aware devices during rendezvousing scenarios mimicking typical real-life situations. We first present the methodology for our study including a description of our Wizard-of-Oz approach to providing location-awareness. We then present the results of this work, focusing on one of the rendezvous scenarios. Finally, we reflect on the results, providing insights into the differences between mobile phone and location-aware device usage and their impact on users' behaviours.

\section{RENDEZVOUS FIELD STUDY}

\section{Participants \& Setting}

Forty-eight participants (28 male and 20 female) took part in this field study. The study took place in July 2004, in a busy downtown shopping area.

\section{Experimental Conditions}

Participants took part in one of three technology conditions.

In the mobile phone condition, both participants were given a mobile phone programmed with their partner's phone number and a laminated paper map that showed most of the buildings in the area (without names).

In the location-aware handheld condition participants were provided with an HP iPAQ h4155 handheld computer. Each handheld ran custom location-awareness software that enabled participants to view a street map of the area (identical to the paper map) annotated with the participants' locations as well as the rendezvous location (see Figure 1). Different coloured dots on the map represent each participant. Approximately $1 / 6$ of the map was visible at a time and participants panned the display to see the rest. The software also provided participants with the ability to request a rendezvous location. Participants selected the rendezvous icon, moved it to the desired location (mirrored on their partner's screen), and then selected the 'ask' option from the rendezvous menu at the bottom of the screen. This caused a request message to pop up on their partner's screen. The partner responded by accepting, rejecting or 


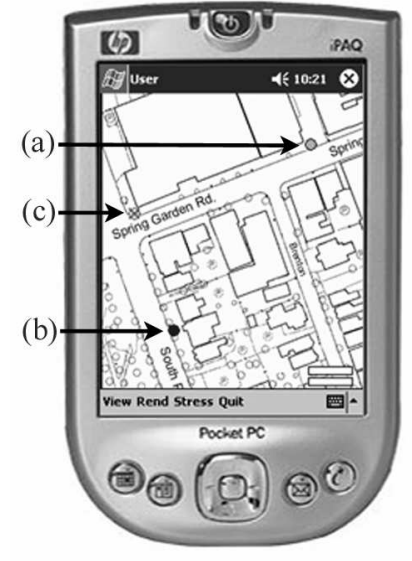

Figure 1. Interface for the location-aware device. (a) Partner's dot; (b) participant's dot; (c) rendezvous ' $X$ '.

ignoring the request. The rendezvous ' $\mathrm{X}$ ' turned green when the location was agreed upon.

In the mobile phone and location-aware handheld condition, participants were provided with both a mobile phone and the handheld. The participants were free to use either device at any time during the study.

\section{Wizard of Oz Approach to Location-Awareness}

The GPS technology to which we had access could not reliably provide continuous location-awareness information given our study environment and public Wi-Fi access was not available. We therefore chose to simulate locationawareness and wireless connectivity with a Wizard of $\mathrm{Oz}$ approach. Two "Wizards" were each assigned a participant to track and walked a short distance behind that participant (see Figure 2). A Bluetooth connection between each participant's handheld and the corresponding Wizard's handheld allowed the Wizards to update the participant's handheld. The two Wizards themselves were in constant contact via 2-way radios, communicating the location of the participant they were following, along with any rendezvous requests or acknowledgements.

\section{Rendezvous Scenarios}

The scenarios used were based on three rendezvousing behaviours identified by Colbert [1,2]:

- Arranging a rendezvous while separated

- Negotiating a new rendezvous location when one partner is unresponsive

- One partner is delayed forcing the other to wait

We focus discussion on the third scenario as it highlights many of the observed changes in behaviour.

\section{Procedure}

At the beginning of each session, the researchers met the participants in a small park located at the edge of the study area. After filling out a background questionnaire, participants were given an introduction to the technology used in their condition (either a mobile phone, a location-

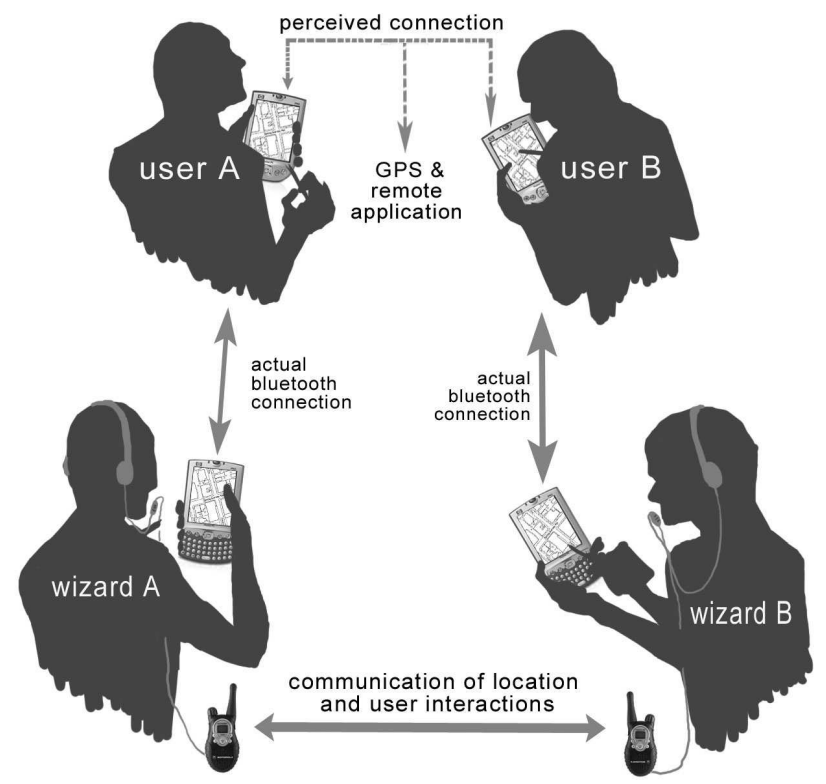

Figure 2. Wizard-of-Oz approach to providing locationawareness.

aware handheld, or both). To ensure that the participants were familiar with the devices and software, they completed a practice rendezvous. Given that the study environment was a high traffic area (both in terms of pedestrians and vehicles) participants were instructed not to run and to obey all local traffic laws.

After the practice rendezvous, participants were informed they would be taking part in three different scenarios where they must meet up with their partner after completing individual tasks designed to separate the participants. Once the individual tasks were completed, the participants were required to negotiate a rendezvous location or meet up at a predefined location and time. The goal for all three scenarios was successful completion of the rendezvous with their partner. After completion of the scenarios, participants took part in a semi-structured interview.

\section{Data Collection \& Analysis}

Data was collected via field notes, audio recordings, data logging, questionnaires, and semi-structured interviews. Pertinent data from these sources was aggregated into a single, linear narrative, enabling us to understand how participants proceeded, given the device condition. Observers following the participants made field notes, recording participants' actions and verbal comments. Each participant was equipped with a voice recorder to create a digital audio recording of all comments and conversations. Data logging captured all actions performed using the location-aware handhelds allowing for precise analysis of selected rendezvous locations and user interactions. Questionnaires gathered background information on participants and participants' perceptions pertaining to the ease of the rendezvous just completed. Finally, post-session semi-structured interviews further probed the participants' 


\begin{tabular}{|c|c|c|}
\hline $\begin{array}{l}\text { Laura arrived first at London Hair Design (the rendezvous } \\
\text { location), one minute before the targeted time. Four } \\
\text { minutes later when Vanessa still hadn't arrived, Laura took } \\
\text { out her mobile phone and called Vanessa. } \\
\text { L: "Hello." } \\
V: \quad \text { "Hello." } \\
\text { L: "Hi. Where are you?" } \\
V: \quad \text { "I am trying to find Curry Village. Brenton St. I can't } \\
\quad \text { find it. Where are you now?" } \\
\text { L: "I am at South Park. London Hair Design. I'm } \\
\quad \text { waiting for you." } \\
\text { V: "So you made it. Ok. I'll be there in about five } \\
\quad \text { minutes." } \\
\text { L: "Ok. Goodbye." } \\
\text { Laura continued to wait until Vanessa arrived three } \\
\text { minutes later. }\end{array}$ & $\begin{array}{l}\text { Emma arrived first at the rendezvous } \\
\text { location, on time. She checked her handheld } \\
\text { computer to see where Natasha was. "Uh oh. } \\
\text { Where is she going?" Emma looked up and } \\
\text { down the street and frequently looked down } \\
\text { at the handheld. Emma started making noises } \\
\text { "Whoa whoa whooooa") as Natasha } \\
\text { appeared to be going the wrong way. Emma } \\
\text { suggested a new rendezvous location on the } \\
\text { corner of South Park St. and Brenton Place. } \\
\text { She indicated that she wanted a quick } \\
\text { rendezvous. She began to walk toward the } \\
\text { new rendezvous location and saw Natasha } \\
\text { approaching. They met up and walked to the } \\
\text { final rendezvous location together. }\end{array}$ & $\begin{array}{l}\text { Jessie arrived first at the rendezvous } \\
\text { location, right on time. She observed her } \\
\text { partner getting closer on the handheld. The } \\
\text { next time she looked at the handheld her } \\
\text { partner's location-indicator was no longer } \\
\text { moving. Jessie picked up the mobile phone } \\
\text { and called Sandy. } \\
\text { J: "Hi. Are you still coming?" } \\
\text { S: "Hello. Hi. At some point. I have to } \\
\quad \quad \text { count pennies first." } \\
\text { J: "Ohhh, ok. Have fun." } \\
\text { S: "Ok, I will." } \\
\text { J: "Call me if anything changes." } \\
\text { S: "Alright. Bye." } \\
\text { Jessie waited and shortly afterward Sandy } \\
\text { arrived. }\end{array}$ \\
\hline
\end{tabular}

Table 1: Scenario 3 narratives: Condition 1: Mobile Phones (left), Condition 2: Location-Aware Handhelds (middle) and Condition 3: Both (right)

rendezvousing experience. Questions were designed to identify participants' choices in given situations and explore the effect of technology on their actions.

There are limitations to the types of data easily collected in the field. Observers trailing participants were unable to count many interactions (e.g. map glances) precisely, so instead recorded more general user behaviours. In addition, the dynamic nature of the field environment and the individual differences of users (familiarity with each other and with the study area) combined to make timing information for the scenarios highly variable. Furthermore, the scenarios we set up did not always happen as planned (e.g. the participant meant to be late sometimes arrived first). As such, we report our results in a narrative manner.

\section{RENDEZVOUS OUTCOMES}

This section characterizes common trends observed for the third rendezvous scenario in each of the three experimental conditions through narratives (see Table 1) and associated discussion. All of the narratives represent real participant data collected during the study. The goal of this scenario was to force one partner to be late for the rendezvous and observe what the waiting partner would do. Participants were sent on individual tasks and given a time ( 7 minutes) and place (London Hair Design) to rendezvous. Once enroute, one of the participants was given a secondary task (count a bag of pennies at Curry Village) making it difficult for them to arrive at the rendezvous location on time. We observed the behaviours of both the waiting participant and the delayed participant including how they made use of the technology and whether or not the waiting participant chose to stay at the rendezvous location.

\section{Condition 1: Mobile Phone}

Three participants chose to call and check in when their partner was late for the rendezvous. All inquired where their partner was and why they were delayed. Two other participants chose to call to let their partner know they were running late. Interestingly, in both cases the caller was not the partner we intentionally delayed but was late because of navigational errors they committed. The participants who were delayed for reasons outside of their control (i.e. told to count pennies) did not choose to call their partners.

For the remaining three pairs, no calls were made. In the post-session interview with these pairs, two indicated that if the wait-time had been longer, they would have called their partner. A third indicated he would have called if he knew his partner was waiting at the rendezvous location.

None of the participants left the rendezvous location to find their partner. One participant continually looked down the street trying to see their partner approaching; however, they were looking down the wrong street. As a result, they were unaware of their partner approaching in the other direction.

\section{Condition 2: Location-Aware Handheld}

All participants who arrived first made use of the locationawareness information while waiting. Upon arrival at the rendezvous location, they immediately checked their handheld to determine the location of their partner. These participants continued to monitor the progress of their partner until they made visual contact. In four instances, the person waiting at the rendezvous location chose to walk toward their partner's location. The remainder of the pairs waited at the rendezvous location for their partner to arrive.

Besides general concern over their partner being late, the location-awareness information did contribute to some uncertainty and confusion when the partner's locationindicator wasn't moving (while they were counting pennies). One participant said she was frustrated that her partner was stopped and she wanted to tell him to move up.

\section{Condition 3: Both Mobile Phone and Handheld}

Again, all participants who arrived first utilized the location-awareness information and immediately checked their handheld to determine their partner's location. Four pairs chose to additionally communicate with their partner with the mobile phone. In three cases, the waiting participant placed a call to inquire where their partner was and why they were delayed. In the fourth case, the delayed participant called his partner to say he was running late and would arrive shortly. The remaining pairs simply monitored their partner's movements with the handheld and did not 
use the mobile phones to communicate with their partner. None of the waiting participants left the rendezvous location to attempt to meet up with their partner sooner.

\section{DISCUSSION}

Regardless of the technology provided to the participants, all of the pairs were able to complete the rendezvous tasks without much difficulty. However, the results of this study clearly demonstrate that the participants exhibited very different behaviours depending on the technology used.

Mobile phones are an easy medium to assist people in communicating information about actions and intentions (i.e. 'what are you are doing?' or 'where are you planning to go?'). This information can be difficult to gather from sensor-based devices such as location-aware handhelds. In contrast, sensor-based devices are very good at gathering overt contextual information, such as location, in a very unobtrusive manner. However, they provide little assistance in interpreting the associated state of the person. In our study, when participants were given both devices, they easily recognized the strengths of each device and utilized each appropriately (i.e. monitoring their partner's location with the handheld and using the mobile phone to call when they were confused about what the person was doing).

The amount and type of information available to people can influence their rendezvousing behaviour. In the mobile phone condition, when one partner was waiting for the other, none chose to leave the rendezvous location in an attempt to meet their partner. This is not surprising as, without location information, they may not have known where their partner was. Even if they used the mobile phone to determine their partner's location, it would still have been difficult to infer the direction in which their partner would proceed in order to intercept them.

In the location-aware handheld condition, half of the participants chose to leave the rendezvous location to attempt to meet their partner. Being aware of their partner's location allowed them to easily find (and intercept) their partner. However, in the final condition when the participants had access to both devices, none of the participants chose to leave. This suggests that the reason the participants left the rendezvous location in the locationaware handheld condition was more a result of missing contextual information rather than the ease with which they could meet up with their partner. Participants who chose to leave seemed confused about their partner's actions or believed they were lost. In contrast, in the final condition, the participants used the mobile phone to call their partner and gather this information. This potentially gave them a better understanding of how their partner was proceeding, allowing them to make a more informed decision as to how the rendezvous was progressing. All of the delayed participants in the final condition indicated they would be at the rendezvous location shortly so none of their partners waiting at the rendezvous felt compelled to leave.
Before running this study, we felt that location-awareness information would always be beneficial to people attempting to rendezvous. In our third scenario, we observed instances where location-awareness information was extremely beneficial and other instances where it was detrimental. It was beneficial because participants could see their partner's state (location and movement) unobtrusively. This arguably provided the waiting partner with enough information to wait contently. However, when their partner appeared to be lost or not making progress, it was very disconcerting to the waiting partner because they didn't have enough contextual information to determine what the problem was. This uncertainty was strong enough in some cases to actually draw the waiting partner away from the rendezvous location.

\section{CONCLUSION}

The observations gathered in our study clearly demonstrate that the type of technology provided to people significantly impacts their rendezvous behaviour. Both state and context are essential pieces of information. However, locationbased devices and mobile phones represent opposite ends of the spectrum. It is important to investigate additional approaches to gain contextual and state information in location-aware computing.

\section{ACKNOWLEDGMENTS}

Thanks to the members of the EDGE Lab for their support, particularly Mike and Vicki who played the role of Wizards. Funding was provided in part by NSERC.

\section{REFERENCES}

1. Colbert, M. (2001). A Diary Study of Rendezvousing: Implications for Position-Aware Computing and Communications for the General Public. In Proceedings of GROUP 2001, Boulder, CO. 15-23.

2. Colbert, M. (2002). A Diary Study of Rendezvousing: Group Size, Time Pressure and Connectivity. In Proceedings of Mobile HCI 2002, Pisa, Italy.

3. Griswold, W. G., Boyer, R., Brown, S. W., Truong, T. M., Bhasker, E., Jay, G. R., et al. (2003).

Activecampus - Experiments in Community-Oriented

Ubiquitous Computing (No. Technical Report CS2003-0765). UC San Diego.

4. Ito, M. and Okabe, D. (2005). Technosocial Situations: Emergent Structuring of Mobile Email Use. In Misa Matsuda (Ed.), Personal, Portable, Pedestrian: Mobile Phones in Japanese Life. Cambridge: MIT Press.

5. Marmasse, N., Schmandt, C., and Spectre, D. (2004). Watchme: Communication and Awareness between Members of a Closely-Knit Group. In Proceedings of UbiComp, Nottingham, UK.

6. Pousman, Z., Iachello, G., Fithian, R., Moghazy, J., and Stasko, J. (2004). Design Iterations for a Location-Aware Event Planner. Personal Ubiquitous Computing, 8(2): 117-125. 